\title{
Large deviations of lattice Hamiltonian dynamics coupled to stochastic thermostats.
}

\author{
Thierry Bodineau*and Raphaël Lefevere ${ }^{\dagger}$
}

November 13, 2018

\begin{abstract}
We discuss the Donsker-Varadhan theory of large deviations in the framework of Hamiltonian systems thermostated by a Gaussian stochastic coupling. We derive a general formula for the Donsker-Varadhan large deviation functional for dynamics which satisfy natural properties under time reversal. Next, we discuss the characterization of the stationary states as the solution of a variational principle and its relation to the minimum entropy production principle. Finally, we compute the large deviation functional of the current in the case of a harmonic chain thermostated by a Gaussian stochastic coupling.
\end{abstract}

\section{Introduction.}

In the recent years, several studies of large systems out of equilibrium through fluctuation theory have been made [2, 3, 4, 5, 16, 18, 19, 20]. In a recent series of papers [28, 8, 29, 30, 31], it has been understood that in random systems driven out of equilibrium, the theory of large deviations provides naturally a variational characterization of the steady states which is related to the minimum entropy production principle. In this paper, we pursue this approach in the framework of thermostated lattices of Hamiltonian oscillators and investigate in this setting the Donsker-Varadhan large deviation theory [11, 12]. The existence of a large deviation principle for a chain of Hamiltonian oscillators has been already established in [39, 35], here we focus on the physical interpretation of the functional in terms of entropy production and on exact computations for Gaussian dynamics.

Thermostated lattices of Hamiltonian oscillators arise naturally in physics as they model either the contact with an environment, the nonlinearity of the dynamics or the randomness of initial conditions by effective stochastic Gaussian terms added to Hamilton's equations of motion. For the class of models considered in this paper and introduced in section 2, the noise is degenerate as it acts only on some coordinates (the momenta). Building on [24], we introduce dynamics with a deterministic driving force which are analogous to the asymmetric dynamics in lattice gas models. These models satisfy the generalized detailed balance which is a relation

${ }^{*}$ Ecole Normale Supérieure, DMA, 45 rue d'Ulm 75230 PARIS Cedex 05, FRANCE

${ }^{\dagger}$ LPMA, Université Denis Diderot (P7) - Boîte courrier 7012, 75251 PARIS Cedex 05, FRANCE 
between the generator of the dynamics, a reference measure and a time-reversal breaking function that is a linear combination of the local energy currents.

In section 3, we rephrase the Donsker-Varadhan theory in our setting [39] and establish a useful formula (41) for the large deviation functional of processes which have natural properties under time reversal. This generalizes the formula expressing the functional as the Dirichlet form of the process in the case of reversible dynamics.

In section 3.2, we discuss, along the lines of [28, 29, 30, 31], the characterization of the stationary measures out of equilibrium in terms of a variational principle and we stress the differences and the peculiarities inherent to the Hamiltonian structure. In particular, Hamiltonian dynamics leaves the Gibbs (or Shannon) entropy invariant, thus it comes as no surprise that the so-called minimum (Gibbs) entropy production principle fails to determine correctly stationary states in systems out of equilibrium or even in equilibrium: it is blind to the Hamiltonian dynamics. The only part of the dynamics modifying entropy is the diffusion modeling the action of the thermostats which, in our case, act only on the momenta variables. The degeneracy of the dynamics (non-ellipticity) is the main reason for which the minimum entropy production fails to select the correct stationary state. We remark that in [8] (section 3.3), this principle was shown to be valid in the case of an Hamiltonian system coupled to a diffusion acting on all the variables. We analyze the interplay between the entropy producing part of the dynamics and the purely Hamiltonian part and show that the Donsker-Varadhan functional provides a variational characterization of the stationary measure (both in equilibrium and out of equilibrium) related to the minimum entropy production principle.

Finally in section 3.4, we recover from the symmetries of the Donsker-Varadhan functional the Gallavotti-Cohen symmetry [18, 19, 16, 21, 22, 23, 26, 14, 35.

In section 4, we focus on the large deviations of the heat current for thermostated lattices of harmonic oscillators. We compute exactly the functional and relate it to previous expressions derived for lattice gas models [3, 4, 5].

\section{Models}

We will first recall the general framework of Hamiltonian dynamics and define the relevant physical quantities in this context. In section 2.2 , the notion of generalized detailed balance is introduced. It will be an important feature of the Hamiltonian systems with a stochastic forcing considered in this paper (see sections 2.3, 2.4 and 2.5).

\subsection{Hamiltonian dynamics}

We consider a one-dimensional lattice of $N$ particles $(\underline{q}, \underline{p})=\left(q_{i}, p_{i}\right)_{1 \leq i \leq N}$, each one moving around an equilibrium position, the position of the $i$-th particle is denoted by $q_{i}$ and its momentum by $p_{i}$. The systems we have in mind are described by a Hamiltonian, which is the energy function of the set of particles,

$$
H(\underline{q}, \underline{p})=\sum_{i=1}^{N}\left[\frac{p_{i}^{2}}{2}+V\left(q_{i}\right)+\frac{1}{2}\left(U\left(q_{i+1}-q_{i}\right)+U\left(q_{i}-q_{i-1}\right)\right)\right] .
$$


We will consider either periodic boundary conditions (with the convention $q_{N+1}=q_{1}$ and $q_{0}=q_{N}$ ) or open systems with the convention $q_{0}=q_{N+1}=0 . \quad V$ is the potential energy corresponding to an interaction with an external substrate. $U$ describes the potential energy of the interaction between nearest-neighbours. Precise assumptions on the growth of the potentials will be detailed in Section [3.1] along with the mathematical statements.

The positions $q_{i}$ and momenta $p_{i}$ of the particles obey the Hamilton's equations,

$$
\dot{q}_{i}=\frac{\partial H}{\partial p_{i}}, \quad \dot{p}_{i}=-\frac{\partial H}{\partial q_{i}} .
$$

The generator of the Hamiltonian dynamics is given by

$$
L_{H}=\sum_{i=1}^{N}-\frac{\partial H}{\partial q_{i}} \frac{\partial}{\partial p_{i}}+\frac{\partial H}{\partial p_{i}} \frac{\partial}{\partial q_{i}} .
$$

In order to describe the propagation of heat in the lattice, one defines a local energy function,

$$
h_{i}(\underline{p}, \underline{q})=\frac{p_{i}^{2}}{2}+V\left(q_{i}\right)+\frac{1}{2}\left(U\left(q_{i}-q_{i+1}\right)+U\left(q_{i-1}-q_{i}\right)\right),
$$

such that $H=\sum_{i=1}^{N} h_{i}$. The local energy transfer is identified with the transfer of mechanical energy between nearest-neighbours. The energy current is therefore defined through the time evolution of the local energy,

$$
\frac{d h_{i}}{d t}=L_{H} h_{i}=j_{i}-j_{i-1}
$$

with $j_{i}$ the microscopic current of energy or heat between oscillator $i$ and $i+1$

$$
j_{i}=-\frac{1}{2} U^{\prime}\left(q_{i}-q_{i+1}\right)\left(p_{i}+p_{i+1}\right) \text {. }
$$

In section 4, we will investigate the large deviations of the spatial average of the current defined by

$$
J(\underline{q}, \underline{p})=\frac{1}{N} \sum_{i=1}^{N} j_{i} .
$$

When such a Hamiltonian system is in thermal equilibrium at a temperature $T=\beta^{-1}$, its statistical properties are described by the Boltzmann-Gibbs probability distribution over the phase space $\mathbf{R}^{2 N}$,

$$
\rho(\underline{p}, \underline{q})=\frac{1}{Z} \exp \left(-\frac{1}{T} H(\underline{p}, \underline{q})\right) .
$$

Remark that with respect to that distribution, $\left\langle p_{i}^{2}\right\rangle=T$ and the averaged current is null $\left\langle j_{i}\right\rangle=0$. This last identity is straightforward because the equilibrium distribution is even under the reversal of momenta $\underline{p} \rightarrow-\underline{p}$ while the current is odd. 


\subsection{The generalized detailed balance}

We now recall the notion of reversibility in the framework of lattices of coupled oscillators. Then, we introduce the notion of generalized detailed balance which will apply when the chain of oscillators is forced out of equilibrium by stochastic heat baths (sections 2.3 and 2.4) or a driving force (section 2.5).

In Hamiltonian dynamics, the variables $\underline{p}$ basically describe the velocities of the particles and are therefore odd functions under time reversal. When isolated, those systems are reversible in the sense that if one lets evolve the particles from some initial conditions at time 0 up to some time $T$ and then reverse all momenta, Hamilton's dynamics will take back the particles through the same trajectory but with reverse momenta. Thus in the case of Hamiltonian dynamics (with possibly a stochastic forcing), the standard notion of detailed balance has to take into account the reversal of momenta. We denote by $\Pi$ the operator which reverses momenta

$$
\Pi(\underline{q}, \underline{p})=(\underline{q},-\underline{p})
$$

Let $P_{t}\left((\underline{q}, \underline{p}),\left(\underline{q}^{\prime}, \underline{p}^{\prime}\right)\right)$ be the semi-group associated to an Hamiltonian dynamics with a stochastic perturbation (for a precise definition see sections 2.3 and 2.4 ) with initial data $(\underline{q}, \underline{p})$ and final data $\left(\underline{q}^{\prime}, \underline{p}^{\prime}\right)$ at time $t$. We consider a probability measure with density $\rho(\underline{q}, \underline{p})$ symmetric wrt to time reversal $\Pi \rho=\rho$. The dynamics is reversible wrt to the density $\rho(\underline{q}, \underline{p})$ if for any time $t$

$$
\rho(\underline{q}, \underline{p}) P_{t}\left((\underline{q}, \underline{p}),\left(\underline{q}^{\prime}, \underline{p}^{\prime}\right)\right)=\rho\left(\underline{q}^{\prime}, \underline{p}^{\prime}\right) P_{t}\left(\Pi\left(\underline{q}^{\prime}, \underline{p}^{\prime}\right), \Pi(\underline{q}, \underline{p})\right) \text {. }
$$

Let $L$ be the generator of this dynamics and let $L^{\dagger}$ denote the adjoint with respect to the Lebesgue measure. The adjoint $L_{\rho}^{*}$ of the operator $L$ with respect to the measure with density $\rho(\underline{q}, \underline{p})$ is defined by

$$
L_{\rho}^{*} f(\underline{q}, \underline{p})=\rho^{-1}(\underline{q}, \underline{p}) L^{\dagger}(\rho(\underline{q}, \underline{p}) f(\underline{q}, \underline{p}))
$$

for any regular function $f(\underline{q}, \underline{p})$. Alternatively, $L_{\rho}^{*}$ satisfies

$$
\langle f L g\rangle_{\rho}=\left\langle g L_{\rho}^{*} f\right\rangle_{\rho} .
$$

$L_{\rho}^{*}$ is therefore interpreted as the generator of the time reversed dynamics sampled with initial data distributed according to $\rho$. Applying (10) for an infinitesimal amount of time, the equivalent form of the detailed balance relation can be obtained

$$
L_{\rho}^{*}=\Pi L \Pi,
$$

where we used that the density $\rho$ satisfies $\Pi \rho=\rho$. For stochastic dynamics, the usual detailed balance relation does not involve the time reversal operator, however we keep the same terminology for simplicity.

For general Hamiltonian systems coupled to stochastic thermostats, the reversibility may not hold and (12) has to be replaced by the generalized detailed balance relation (see for example [14]) which can be defined as follows 
Definition 1 We consider an evolution with generator $L$ and $\rho(\underline{q}, \underline{p})$ a reference measure such that $\Pi \rho=\rho$. If there exists a function $\sigma(\underline{q}, \underline{p})$ such that

$$
L_{\rho}^{*}=\Pi L \Pi+\sigma, \quad \text { and } \quad \Pi \sigma=-\sigma,
$$

then the triplet $(L, \rho, \sigma)$ is said to satisfy a generalized detailed balance relation. By convention in (13), $\sigma$ acts as a multiplication operator.

This definition will be illustrated in the examples introduced in sections 2.3, 2.4 and 2.5. In equilibrium dynamics (when $\sigma=0$ ) and when sampled with initial conditions distributed according to the equilibrium measure, the dynamics is equivalent to its time-reverse, this is the detailed balance principle. When the system is subject to some non-equilibrium dynamics forcing a heat current through the system, the stationary state looses its invariance under time-reversal $(\sigma \neq 0)$. In Markovian systems, the generalized detailed balance is a central feature of the Gallavotti-Cohen symmetry [26, 16, 18, 21, 23, 34, 35] for the large deviation functional of the function $\sigma$ (see section 3.4).

Typically $\sigma$ is a function of the microscopic currents of energy in the lattice and the reference measure is an equilibrium or a local equilibrium distribution. A given dynamics may satisfy a generalized detailed balance relation with respect to different reference measures and different functions $\sigma$ (see section 2.4).

\subsection{Heat in the bulk}

The simplest perturbation of the Hamiltonian dynamics (2) is to couple each oscillator to a heat bath. The boundary conditions are fixed $q_{0}=q_{N+1}=0$ and each oscillator $i=1, \ldots, N$ evolves according to

$$
\begin{aligned}
& d q_{i}=p_{i} d t \\
& d p_{i}=-\gamma_{i} p_{i} d t-\frac{\partial H}{\partial q_{i}} d t+\sqrt{2 \gamma_{i} T_{i}} d w_{i},
\end{aligned}
$$

where the $w_{i}$ are standard independent Brownian motions, $T_{i}=\beta_{i}^{-1}$ is the temperature of each heat bath and $\gamma_{i}>0$ is the friction. The generator of the dynamics is given by

$$
L=L_{S}+L_{H}
$$

where the Hamiltonian part $L_{H}$ was introduced in (3) and the symmetric part is

$$
L_{S}=\sum_{i=1}^{N}-\gamma_{i} p_{i} \frac{\partial}{\partial p_{i}}+\gamma_{i} T_{i} \frac{\partial^{2}}{\partial p_{i}^{2}}
$$

When the temperatures $\left\{T_{i}\right\}_{i}$ are not equal, the reversibility is lost and the invariant measure unknown. The self-consistent chain [6], for which the temperatures $\left\{T_{i}\right\}_{i}$ are tuned in order to maintain a constant average microscopic current along the chain, falls in the framework of the dynamics (14).

We check now that this dynamics satisfies the generalized detailed balance (see definition (1). Given a collection of inverse temperatures $\underline{\beta}=\left(\beta_{1}, \ldots, \beta_{N}\right)$, we take as a reference measure the following density

$$
\rho_{\underline{\beta}}(\underline{p}, \underline{q})=\frac{1}{Z} \exp (-\hat{H}(\underline{p}, \underline{q})), \quad \text { with } \quad \hat{H}(\underline{p}, \underline{q})=\sum_{i=1}^{N} \beta_{i} h_{i}(\underline{p}, \underline{q}),
$$


where $h_{i}$ are the local energies introduced in (44) and $\beta_{i}^{-1}=T_{i}$ is the temperature of the heat bath at site $i$. We compute now $L_{\rho_{\beta}}^{*}$ the adjoint of $L$ with respect to the measure $\rho_{\underline{\beta}}$. Since $L_{S}=\Pi L_{S} \Pi$ and the stochästic part of the dynamics is reversible with respect to the measure $\rho_{\beta}$, one has for any function $f$

$$
L_{S} f(\underline{q}, \underline{p})=\rho_{\underline{\beta}}^{-1}(\underline{q}, \underline{p}) L_{S}^{\dagger}\left(\rho_{\underline{\beta}}(\underline{q}, \underline{p}) f(\underline{q}, \underline{p})\right) .
$$

We turn now to the Hamiltonian part. Thanks to the relation $L_{H}^{\dagger}=-L_{H}$, we get

$$
\rho_{\underline{\beta}}^{-1} L_{H}^{\dagger} \rho_{\underline{\beta}}=\sum_{i=1}^{N} \beta_{i} L_{H} h_{i}
$$

From (5), one has $L_{H} h_{i}=j_{i}-j_{i-1}$ with the convention $j_{0}=j_{N}=0$, thus

$$
\rho_{\underline{\beta}}^{-1} L_{H}^{\dagger} \rho_{\underline{\beta}}=\sum_{i=1}^{N} \beta_{i}\left(j_{i}-j_{i-1}\right)=-\sum_{i=1}^{N-1}\left(\beta_{i+1}-\beta_{i}\right) j_{i} .
$$

Since $\Pi L_{H} \Pi=-L_{H}=L_{H}^{\dagger}$, this implies that

$$
L_{\underline{\rho^{\beta}}}^{*}=\Pi L \Pi+\sigma_{\underline{\beta}}, \quad \text { with } \quad \sigma_{\underline{\beta}}=-\sum_{i=1}^{N-1}\left(\beta_{i+1}-\beta_{i}\right) j_{i} .
$$

Thus the triplet $\left(L, \rho_{\underline{\beta}}, \sigma_{\underline{\beta}}\right)$ satisfies a generalized detailed balance relation and $\sigma_{\underline{\beta}}$ is a linear combination of the local currents.

\subsection{Heat at the boundary}

If there are no heat baths in the bulk $\left(\gamma_{i}=0\right.$ for $\left.i=2, \ldots, N-1\right)$, the dynamics (14) represents a crystal of atoms heated at two different temperatures at its boundaries. The equations of motion are given by,

$$
\begin{aligned}
d q_{i} & =p_{i} d t, \quad i=1, \ldots, N \\
d p_{i} & =-\frac{\partial H}{\partial q_{i}}(\underline{p}, \underline{q}) d t, \quad i=2, \ldots, N-1
\end{aligned}
$$

and,

$$
\begin{aligned}
d p_{1} & =-\frac{\partial H}{\partial q_{1}}(\underline{p}, \underline{q}) d t-\gamma p_{1} d t+\sqrt{2 \gamma T_{1}} d w_{1}, \\
d p_{N} & =-\frac{\partial H}{\partial q_{N}}(\underline{p}, \underline{q}) d t-\gamma p_{N} d t+\sqrt{2 \gamma T_{N}} d w_{N},
\end{aligned}
$$

where $T_{1}$ and $T_{N}$ stand for the temperature of the left and right reservoirs, respectively, whereas $w_{1}$ and $w_{N}$ are two independent standard Wiener processes.

When $T_{1}=T_{N}=T=\beta^{-1}$, the Gibbs measure (8) is invariant (stationary) for the stochastic dynamics defined above. For two different temperatures, existence, uniqueness and exponential convergence to an unique invariant state has been established under fairly general conditions on the potentials $U$ and $V[9,13,15,34$. 
A computation similar to the case of heat baths in the bulk (21) shows that the dynamics (22) satisfies the generalized detailed balance (see definition 10) for any reference measure $\rho_{\underline{\beta}}$ of the form (17)

$$
L_{\rho_{\underline{\beta}}}^{*}=\Pi L \Pi+\sigma_{\underline{\beta}}, \quad \text { with } \quad \sigma_{\underline{\beta}}=-\sum_{i=1}^{N-1}\left(\beta_{i+1}-\beta_{i}\right) j_{i},
$$

provided the collection of inverse temperatures $\underline{\beta}=\left(\beta_{1}, \ldots, \beta_{N}\right)$ is such that $\beta_{1}^{-1}=$ $T_{1}$ and $\beta_{N}^{-1}=T_{N}$. Thus any $\sigma_{\beta}$ such that $\beta_{1}^{-1}=T_{1}$ and $\beta_{N}^{-1}=T_{N}$ will satisfy the Gallavotti-Cohen symmetry relation (see section 3.4). This was already observed in [14, 34].

\subsection{Asymmetric periodic chain}

Building on a previous work [24, we introduce new dynamics by adding a mechanical force which creates a current through the system. On a periodic lattice, these dynamics lead to non-equilibrium systems with non-vanishing currents and in this sense, they are reminiscent of the asymmetric processes in lattice gas dynamics [36].

We start with an heuristic discussion before giving the definition of the dynamics. If the heat baths are at different temperatures, the dynamics (22) is no longer reversible with respect to the Gibbs measure or any "local equilibrium" measure $\rho_{\beta}$. As observed in (24), the function $\sigma$ which breaks the reversibility is a linear combination of the local energy currents. We show that adding an appropriate mechanical force allows to modify at will the coefficients of this linear combination. In particular, the reversibility may be restored in (22) by tuning the intensity of the additional mechanical force. From the point of view of the generalized detailed balance, the action of the mechanical force is equivalent to the action of the local temperature gradient.

Let us first show that one may choose a (non-Hamiltonian) force which modifies the coefficients of the combination of local currents of energy in $\sigma$. Take as generator of the dynamics,

$$
L=L_{S}+L_{H}+L_{\underline{\theta}},
$$

where $L_{H}$ is the generator of the Hamiltonian dynamics (3),$L_{S}$ the generator of the two stochastic reservoirs (23)

$$
L_{S}=-\gamma p_{1} \frac{\partial}{\partial p_{1}}+\gamma T_{1} \frac{\partial^{2}}{\partial p_{1}^{2}}-\gamma p_{N} \frac{\partial}{\partial p_{N}}+\gamma T_{N} \frac{\partial^{2}}{\partial p_{N}^{2}},
$$

and the contribution of the mechanical drift is given by the antisymmetric operator

$$
L_{\underline{\theta}}=-\sum_{i=1}^{N} \frac{1}{2} T_{i}\left(\theta_{i-1} U^{\prime}\left(q_{i-1}-q_{i}\right)+\theta_{i} U^{\prime}\left(q_{i}-q_{i+1}\right)\right) \frac{\partial}{\partial p_{i}},
$$

To study the reversibility (12) properties of this dynamics, we first compute,

$$
\begin{aligned}
\rho_{\underline{\beta}}^{-1} L_{\underline{\theta}}^{T} \rho_{\underline{\beta}} & =-\sum_{i=1}^{N} \frac{1}{2}\left(\theta_{i-1} U^{\prime}\left(q_{i-1}-q_{i}\right)+\theta_{i} U^{\prime}\left(q_{i}-q_{i+1}\right)\right) p_{i} \\
& =-\sum_{i=1}^{N-1} \theta_{i} \frac{1}{2}\left(p_{i}+p_{i+1}\right) U^{\prime}\left(q_{i}-q_{i+1}\right)=\sum_{i=1}^{N-1} \theta_{i} j_{i} .
\end{aligned}
$$


Since the operator $L_{S}+L_{H}$ satisfies a generalized detailed balance relation wrt $\sigma_{\underline{\beta}}=-\sum_{i=1}^{N-1}\left(\beta_{i+1}-\beta_{i}\right) j_{i}(24)$, we see that the dynamics (25) satisfies now the generalized detailed balance (see definition 1) for the measure $\rho_{\underline{\beta}}$ (17) with respect to $\sigma_{\underline{\beta}, \underline{\theta}}$

$$
L_{\underline{\rho_{\underline{\beta}}}}^{*}=\Pi L \Pi+\sigma_{\underline{\beta}, \underline{\theta}}, \quad \text { with } \quad \sigma_{\underline{\beta}, \underline{\theta}}=\sum_{i=1}^{N-1}\left(\beta_{i}-\beta_{i+1}+\theta_{i}\right) j_{i} .
$$

When the relation $\theta_{i} \neq\left(\beta_{i+1}-\beta_{i}\right)$ for some $i$, then the system is driven out of equilibrium. However, with the choice $\theta_{0}=\theta_{N}=0$ and $\theta_{i}=\left(\beta_{i+1}-\beta_{i}\right)$ reversibility is restored. This is the key point which allows to identify the strength of the mechanical force with the action of the local gradient of temperature.

For $\theta_{i}=\left(\beta_{i+1}-\beta_{i}\right)$ and slowly varying temperatures of the form $\beta_{i}^{-1}=T_{i}=T\left(\frac{i}{N}\right)$ where $T$ is a smooth function, the generator $L_{\underline{\theta}}$ becomes at lowest order in $\frac{1}{N}$,

$$
L_{\underline{\theta}}=\sum_{i=1}^{N} \frac{1}{N} \frac{\nabla T\left(\frac{i}{N}\right)}{2 T\left(\frac{i}{N}\right)}\left(U^{\prime}\left(q_{i-1}-q_{i}\right)+U^{\prime}\left(q_{i}-q_{i+1}\right)\right) \frac{\partial}{\partial p_{i}} .
$$

Note that in the sum over $i$, the prefactors depending on the temperature and its gradient are basically constant when $i$ varies over distances much smaller than $N$.

The asymmetric periodic chain [24], described below, is a chain with periodic boundary conditions and a dynamics made of three parts. The first one corresponds to a usual Langevin dynamics for each oscillator, the second one to the Hamiltonian dynamics on the lattice and the third one is the previous generator (28) with constant prefactors (but arbitrary values). Namely, it is defined as

$$
L=L_{S}+L_{H}+L_{\tau}
$$

with,

$$
L_{S}=\sum_{i}-\gamma p_{i} \frac{\partial}{\partial p_{i}}+\gamma T \frac{\partial^{2}}{\partial p_{i}^{2}}
$$

$L_{H}$ is defined in (3) and the part driving the system out of equilibrium is,

$$
L_{\tau}=-\frac{\tau}{2 T} \sum_{i}\left(U^{\prime}\left(q_{i-1}-q_{i}\right)+U^{\prime}\left(q_{i}-q_{i+1}\right)\right) \frac{\partial}{\partial p_{i}} .
$$

In terms of equations of motion the dynamics is described as follows,

$$
\begin{aligned}
& d q_{i}=p_{i} d t \\
& d p_{i}=-\gamma p_{i} d t-\frac{\partial H}{\partial q_{i}} d t-\frac{\tau}{2 T}\left(U^{\prime}\left(q_{i-1}-q_{i}\right)+U^{\prime}\left(q_{i}-q_{i+1}\right)\right) d t+\sqrt{2 \gamma T} d w_{i}
\end{aligned}
$$

where $\tau \in \mathbf{R}$ is the new parameter regulating the strength of the non-equilibrium forcing and the $w_{i}$ are standard independent Brownian motion $i=1, \ldots, N$. Periodic boundary conditions means $q_{0}=q_{N}$ and $q_{N+1}=q_{1}$. Compared to the dynamics (14), the new term proportional to $\tau$ is the non-equilibrium part of the dynamics. As should be clear from the above argument it is responsible for the breaking of the timereversal symmetry of the equilibrium dynamics $(\tau=0)$. Indeed, for the dynamics (32), taking the Gibbs measure (8) at constant temperature $T$ as the reference 
measure, the generalized detailed balance holds with a function $\sigma$ proportional to the total current (17)

$$
\sigma=\frac{\tau}{T^{2}} \sum_{i=1}^{N} j_{i}=\frac{\tau N}{T^{2}} J
$$

We will show in subsection 3.3 that when $\tau \neq 0, \tau\langle J\rangle_{\hat{\rho}}>0$ for a stationary measure $\hat{\rho}$. Thus, the dynamics ensures the existence of an average non-vanishing energy current in the stationary state. In section 4, the current large deviations for the forced dynamics (32) with harmonic potentials will be computed. We will see there that (32) are the optimal dynamics to realize current deviations.

\section{The large deviation functional}

The goal of this section is to rephrase the Donsker-Varadhan theory [11, 12] in the framework of the Hamiltonian systems coupled to Gaussian stochastic thermostats and to discuss the relation with entropy production.

\subsection{The Donsker-Varadhan functional}

In order to cover all the examples introduced in sections 2.3 and 2.5, we consider the general dynamics defined by

$$
\begin{aligned}
d q_{i} & =p_{i} d t \\
d p_{i} & =-\gamma_{i} p_{i} d t-\frac{\partial H}{\partial q_{i}} d t-\frac{1}{2} T_{i}\left(\theta_{i-1} U^{\prime}\left(q_{i}-q_{i-1}\right)+\theta_{i} U^{\prime}\left(q_{i+1}-q_{i}\right)\right) d t+\sqrt{2 \gamma_{i} T_{i}} d w_{i}
\end{aligned}
$$

with $\gamma_{i}>0, T_{i}>0$ for $i=1, \ldots, N$. We stress the fact that the noise acts at each site $i$. To simplify notation, we restrict to open systems (with the convention $\left.q_{0}=q_{N+1}=0, \theta_{0}=\theta_{N}=0\right)$, but similar results hold with periodic boundary conditions (with the convention $q_{N+1}=q_{1}$ and $q_{0}=q_{N}$ ). We denote by $P$ the probability of the evolution (34) starting from a given initial data (which will play no role in the large $t$ asymptotic).

Typically, we shall be interested in the deviations over time of some physical quantities like the heat current (7). Let the empirical distribution $\nu_{t}$ be defined by,

$$
\nu_{t}(A)=\frac{1}{t} \int_{0}^{t} \mathbf{1}_{A}((\underline{p}(s), \underline{q}(s))) d s,
$$

where $\mathbf{1}_{A}$ is the indicator function of a set $A \subset \mathbf{R}^{2 N}$. If the dynamics is ergodic $\nu_{t}$ converges to the ergodic invariant measure. We now look at the asymptotic probability $P\left[\nu_{t} \simeq \mu\right]$ that the empirical distribution is close to the distribution of a given measure $\mu$ (in the sense of the weak convergence topology [39]) for large $t$. Under suitable hypothesis (see Proposition 1), the dynamics obeys a large deviation principle with rate function $I$ and asymptotically in $t$

$$
P\left[\nu_{t} \simeq \mu\right] \sim \exp (-t I(\mu))
$$

Furthermore, from the Donsker-Varadhan theory [11, 12, 39] the functional is given by

$$
I(\mu)=\sup _{g}\left\{-\left\langle\frac{L g}{g}\right\rangle_{\mu} \mid g \in C_{b}^{\infty}\left(\mathbf{R}^{2 N} ;[1, \infty[)\}\right.\right.
$$


where $C_{b}^{\infty}\left(\mathbf{R}^{2 N} ;[1, \infty[)\right.$ is the set of bounded infinitely differentiable functions in $\mathbf{R}^{2 N}$ taking values larger or equal to 1 . We refer to Lemma 6.3 .7 of [10] for the variational expression of $I$ in the case of hypoelliptic diffusions.

The following Proposition justifies the validity of the large deviation principle (35).

Proposition 1 Suppose that the potentials $V$ and $U$ of the Hamiltonian (1) are convex, twice differentiable and satisfy

$V^{\prime \prime}(q) \geq \delta \quad$ and $\quad \sum_{i=1}^{N} V\left(q_{i}\right)+\frac{U\left(q_{i}-q_{i+1}\right)+U\left(q_{i+1}-q_{i}\right)}{2} \geq \delta \sum_{i=1}^{N}\left(U^{\prime}\left(q_{i}-q_{i+1}\right)\right)^{2}$,

for some constant $\delta>0$.

The dynamics (34) (with $\forall i, \gamma_{i}>0$ ) obeys a large deviation principle with a functional I given by the Donsker-Varadhan theory (36) provided $\max _{i}\left|\theta_{i}\right| \leq \tau_{0}$ and $\max _{i}\left|T_{i}-T_{i+1}\right| \leq \Delta_{0}$, where $\Delta_{0}$ and $\tau_{0}$ are two constants depending only on $\delta,\left\{\gamma_{i}, T_{i}\right\}_{i}$. Furthermore, the previous assumptions on the potentials (37) ensure that the current (7) is exponentially integrable: for any $\lambda$ small enough

$$
\limsup _{t \rightarrow \infty} \frac{1}{t} \log P\left(\exp \left(\lambda \int_{0}^{t} d s J(\underline{p}(s), \underline{q}(s))\right)\right)<\infty .
$$

The proof heavily relies on previous results in the paper [39] and it is postponed to the Appendix. The assumptions of Proposition 1 on the potentials $V$ and $U$ are not optimal, but they are sufficient to cover a wide class of physical examples. In particular, similar statements hold also for any local modifications of the potentials $V$ and $U$. Remark that when the reservoirs act only at the boundary $\left(\gamma_{i}=0, i \in\right.$ $\{2, \ldots, N-1\})$, the large deviation principle for the current has been justified in [35] (for a different class of Hamiltonians).

We are going to rewrite the functional $I$ (36) in a more explicit form. The generator of the dynamics (34) can be decomposed as $L=L_{S}+L_{A}$, with a symmetric part $L_{S}(16)$ due to the noise and an antisymmetric part $L_{A}=\frac{1}{2}(L-\Pi L \Pi)$. We introduce the notation,

$$
\Gamma(f, g)=2 \sum_{i=1}^{N} \gamma_{i} T_{i}\left(\partial_{p_{i}} f\right)\left(\partial_{p_{i}} g\right)
$$

for any smooth functions $f, g$.

Proposition 2 Let $\rho$ be a measure and $\sigma(\underline{q}, \underline{p})$ a function such that

$$
L_{\rho}^{*}=\Pi L \Pi+\sigma .
$$

Let $\mu$ be a measure absolutely continuous with respect to the measure $\rho$, with $f=$ $d \mu / d \rho \in C^{\infty}\left(\mathbf{R}^{2 N} ; \mathbf{R}\right)$ such that $\left\langle\left\|\nabla_{\underline{p}} \sqrt{f}\right\|_{2}\right\rangle_{\rho}<\infty$. Then the functional I (36) is given by

$$
I(\mu)=-\left\langle f^{\frac{1}{2}} L_{S} f^{\frac{1}{2}}\right\rangle_{\rho}+K(\mu)-\frac{1}{2}\langle\sigma\rangle_{\mu},
$$


with

$$
K(\mu)=-\inf _{W}\left(\frac{1}{8}\langle\Gamma(W, W)\rangle_{\mu}+\frac{1}{2}\left\langle L_{A} W\right\rangle_{\mu}\right) \geq 0,
$$

where the infimum is taken over the smooth functions $W \in C^{\infty}\left(\mathbf{R}^{2 N} ; \mathbf{R}\right)$ such that $W \in L^{2}(\mu)$ and $\left|\nabla_{\underline{p}} W\right| \in L^{2}(\mu)$.

From (27), we see that the dynamics (34) satisfies the generalized detailed balance for the measure $\rho_{\underline{\beta}}$ (17), the assumption (40) of Proposition 2 is satisfied with $\rho_{\underline{\beta}}$ and $\sigma_{\underline{\beta}, \underline{\theta}}$

$$
L_{\underline{\rho}_{\underline{\beta}}}^{*}=\Pi L \Pi+\sigma_{\underline{\beta}, \underline{\theta}}, \quad \text { with } \quad \sigma_{\underline{\beta}, \underline{\theta}}=\sum_{i=1}^{N-1}\left(\beta_{i}-\beta_{i+1}+\theta_{i}\right) j_{i} .
$$

Remark that the generalized detailed balance requires symmetry assumptions on the reference measure and on $\sigma$ which are not necessary for the Proposition 2 to hold.

Proof. Let $f=d \mu / d \rho$ and $g \in C_{b}^{\infty}\left(\mathbf{R}^{2 N} ;[1, \infty[)\right.$, then,

$$
\left\langle g^{-1} L g\right\rangle_{\mu}=\left\langle f g^{-1} L g\right\rangle_{\rho}
$$

Write now in (43), $g=\sqrt{h f}$ for some $h>0$ and symmetrize the expression with the help of the adjoint operator of $L$,

$$
\left\langle f g^{-1} L g\right\rangle_{\rho}=\frac{1}{2}\left(\left\langle f^{\frac{1}{2}} h^{-\frac{1}{2}} L\left(h^{\frac{1}{2}} f^{\frac{1}{2}}\right)\right\rangle_{\rho}+\left\langle f^{\frac{1}{2}} h^{\frac{1}{2}} L^{*}\left(h^{-\frac{1}{2}} f^{\frac{1}{2}}\right)\right\rangle_{\rho}\right)
$$

We note the relation,

$$
L(\phi \psi)=\phi L \psi+\psi L \phi+\Gamma(\psi, \phi)
$$

And analogously for $L_{\rho}^{*}=\Pi L \Pi+\sigma=L_{S}-L_{A}+\sigma$ (by hypothesis),

$$
L_{\rho}^{*}(\phi \psi)=\phi\left(L_{S}-L_{A}\right) \psi+\psi\left(L_{S}-L_{A}\right) \phi+\Gamma(\psi, \phi)+\sigma \phi \psi .
$$

Applying those formulas for $L$ (resp. $L_{\rho}^{*}$ ) with $\phi=h^{\frac{1}{2}}$ and $\psi=f^{\frac{1}{2}}$ (resp. $\phi=h^{-\frac{1}{2}}$ and $\psi=f^{\frac{1}{2}}$ ) using the generalized detailed balance relation $L_{\rho}^{*}=\Pi L \Pi+\sigma$ and computing systematically all derivatives, one gets,

$$
\left\langle f g^{-1} L g\right\rangle_{\rho}=\left\langle f^{\frac{1}{2}} L_{S} f^{\frac{1}{2}}\right\rangle_{\rho}+\frac{1}{8}\left\langle\frac{\Gamma(h, h)}{h^{2}}\right\rangle_{\mu}+\frac{1}{2}\left\langle h^{-1} L_{A} h\right\rangle_{\mu}+\frac{1}{2}\langle\sigma\rangle_{\mu} .
$$

Writing $h=e^{W}$ and using the definition (36) of $I(\mu)$, one finally gets the variational formula (42) restricted to functions $W$ such that $(W+\log f) \in C_{b}^{\infty}\left(\mathbf{R}^{2 N} ; \mathbf{R}\right)$. To extend the class of functions $W$ in the variational formula (42), we consider an appropriate sequence of functions $\psi_{n} \in C^{\infty}\left(\mathbf{R}^{2 N} ; \mathbf{R}\right)$ such that $\psi_{n}=1$ in the ball of radius $n$ and $\psi_{n}=0$ outside the ball of radius $n+1$. Then the sequence $W_{n}=$ $W \psi_{n}-\left(1-\psi_{n}\right) \log f$ approximates any function $W \in C^{\infty}\left(\mathbf{R}^{2 N} ; \mathbf{R}\right) \cap L^{2}(\mu)$ with $|\nabla W| \in L^{2}(\mu)$.

The fact that $K(\mu) \geq 0$ follows by choosing $W=0$ in (42). 
One of the important features of the representation (41) for $I(\mu)$ is the presence of $K(\mu)$. As we shall see below, in Proposition 3 and in the example (52), it is in general neither infinite or zero even in the case of dynamics satisfying detailed balance, i.e for equilibrium dynamics. $K(\mu)$ gathers the thermalizing effect of the noise which is transmitted from the $\underline{p}$ variables to $\underline{q}$ variables. It corresponds to the "traffic" in the terminology of [29]. It is invariant under reversal of the sign of the momenta (i.e $K(\mu)=K(\Pi \mu)$ ) and when non-equilibrium forces are included it is invariant under a change of sign of the non-equilibrium parameter as we shall see in the gaussian systems of the last section. It measures the dynamical "activity" in the Hamiltonian system irrespective of the sign of the non-equilibrium parameter. We shall see its role in the variational characterization of stationary states in section 3.2. We now compute a more explicit form for $K(\mu)$.

Proposition 3 Let $\mu$ be a measure on $\mathbf{R}^{2 N}$ with smooth density wrt the Lebesgue measure $d \mu(\underline{p}, \underline{q})=\exp (-\Phi) d \underline{p} d \underline{q}$. If $\bar{W} \in L^{2}(\mu)$ is a solution of

$$
\sum_{i=1}^{N}-\gamma_{i} T_{i} \partial_{p_{i}}^{2} W+\gamma_{i} T_{i} \partial_{p_{i}} \Phi \partial_{p_{i}} W=-L_{A} \Phi,
$$

with $\left|\nabla_{\underline{p}} \bar{W}\right| \in L^{2}(\mu)$ then

$$
K(\mu)=\frac{1}{8}\langle\Gamma(\bar{W}, \bar{W})\rangle_{\mu}=-\frac{1}{4}\left\langle L_{A} \bar{W}\right\rangle_{\mu} .
$$

Moreover, $\langle\Gamma(\bar{W}, \bar{W})\rangle_{\mu}$ is independent from the solution of 48 . Finally, $K$ is symmetric wrt time reversal $K(\Pi \mu)=K(\mu)$.

Proof. Starting from the definition (42) of $K(\mu)$ and integrating by parts, leads to

$$
\frac{1}{8}\langle\Gamma(W, W)\rangle_{\mu}=\frac{1}{4} \sum_{i}-\gamma_{i} T_{i}\left\langle W \partial_{p_{i}}^{2} W\right\rangle_{\mu}+\gamma_{i} T_{i}\left\langle W \partial_{p_{i}} \Phi \partial_{p_{i}} W\right\rangle_{\mu} .
$$

Integrating also by parts the second term in (42) gives,

$$
\frac{1}{2}\left\langle L_{A} W\right\rangle_{\mu}=\frac{1}{2}\left\langle W L_{A} \Phi\right\rangle_{\mu}
$$

Therefore, the solution to the variational problem in (42) gives the equation (48). Using then (50) and (51) and the definition (42) of $K(\mu)$ finally yields (49)). The second equality in (49) follows by combining (50) and (48). To see that $K(\mu)$ does not depend on the solution to (48), let $W, \tilde{W}$ be two different solutions, then,

$$
\sum_{i=1}^{N}-\gamma_{i} T_{i} \partial_{p_{i}}^{2}(W-\tilde{W})+\gamma_{i} T_{i} \partial_{p_{i}} \Phi \partial_{p_{i}}(W-\tilde{W})=0
$$

Multiplying this by $W+\tilde{W}$ and integrating by parts with respect to $\mu$, yields

$$
\langle\Gamma(W+\tilde{W}, W-\tilde{W})\rangle_{\mu}=0,
$$

and thus, $\langle\Gamma(W, W)\rangle_{\mu}=\langle\Gamma(\tilde{W}, \tilde{W})\rangle_{\mu}$. 
The measure $\Pi \mu$ has a density given by $\exp (-\Pi \Phi)$ and $-\Pi \bar{W}$ is the corresponding solution of (48). Thus, the symmetry of $K$ follows from the identity

$$
K(\Pi \mu)=\frac{1}{8}\langle\Gamma(\Pi \bar{W}, \Pi \bar{W})\rangle_{\Pi \mu}=\frac{1}{8}\langle\Gamma(\bar{W}, \bar{W})\rangle_{\mu}=K(\mu) .
$$

This concludes the proof.

Observe also that if $\mu$ is such that $L_{A} \Phi=0$ then $K(\mu)=0$. We want to emphasize that in general $K$ is non-zero and we will illustrate this in the simple case of a one dimensional harmonic oscillator coupled to a heat bath at temperature $T$

$$
L=-\gamma p \frac{\partial}{\partial p}+\gamma T \frac{\partial^{2}}{\partial p^{2}}-\omega^{2} q \frac{\partial}{\partial p}+p \frac{\partial}{\partial q},
$$

with $L_{S}=-\gamma p \frac{\partial}{\partial p}+\gamma T \frac{\partial^{2}}{\partial p^{2}}$ and $L_{A}=-\omega^{2} q \frac{\partial}{\partial p}+p \frac{\partial}{\partial q}$. We will compute $K(\mu)$, where $\mu$ is the Gaussian measure

$$
\mu(q, p)=\frac{1}{\left(2 \pi b \omega^{2}\right)^{\frac{1}{2}}(2 \pi a)^{\frac{1}{2}}} \exp \left(-\left(a \frac{p^{2}}{2}+b \omega^{2} \frac{q^{2}}{2}\right)\right) .
$$

In that case, (48) becomes,

$$
-\gamma T \partial_{p}^{2} W+\gamma T a p \partial_{p} W=\omega^{2} p q(b-a) .
$$

A solution is readily found,

$$
\bar{W}=\frac{b-a}{a \gamma T} \omega^{2} p q
$$

and then, from Proposition 3 ,

$$
K(\mu)=\frac{1}{4 \gamma T}\left(\frac{b-a}{a}\right)^{2} \omega^{4}\left\langle q^{2}\right\rangle_{\mu}=\frac{1}{4 \gamma T}\left(\frac{b-a}{a}\right)^{2} \frac{\omega^{2}}{b} .
$$

From this example, we see that $K(\mu)$ vanishes only for Gaussian measures in which the average potential energy and kinetic energy are equal, i.e when equipartition of energy is realized. In a sense, $K$ measures how much the Gaussian noise acting on the momenta variables $\underline{p}$ is transmitted to the positions variables $\underline{q}$.

\subsection{Entropy production}

In this section, we show that the minimum entropy production principle does not apply for Hamiltonian dynamics and that the large deviation functional (41) is a natural extension for a variational characterization of the steady state. Connections between large deviation functionals in stochastic systems and entropy production were initiated in [28]. In order to identify the average entropy production of a dynamics in a given measure, we proceed as in [36] in the context of interacting particle systems. Namely, we define the entropy production as the difference between the variation of the Gibbs (Shannon) entropy and the transfer of heat by unit time due to the action of the thermostats. A similar computation was performed in [27] for 
heat conduction networks and the identification of the average entropy production with the Dirichlet form of the process was obtained there and in [14].

We consider the dynamics (34) for which the generator is given by $L=L_{S}+L_{A}$ with a symmetric part given by

$$
L_{S}=-\sum_{i=1}^{N}-\gamma_{i} p_{i} \frac{\partial}{\partial p_{i}}+\gamma_{i} T_{i} \frac{\partial^{2}}{\partial p_{i}^{2}} .
$$

We first introduce the entropy production. In thermodynamics, the entropy variation rate, or entropy production is the transfer of energy per unit time divided by the temperature at which the transfer takes place. Therefore, it is natural to define the average (with respect to a given measure $\mu$ ) entropy flux associated with the exchange of energy with the external heat baths as

$$
\sigma_{e x t}(\mu)=\left\langle\tilde{L}_{S} H\right\rangle_{\mu}
$$

where

$$
\tilde{L}_{S}=\sum_{i=1}^{N} \gamma_{i} T_{i}^{-1} p_{i} \frac{\partial}{\partial p_{i}}+\sum_{i=1}^{N} \gamma_{i} \frac{\partial^{2}}{\partial p_{i}^{2}} .
$$

We have simply divided the contribution of each bath by its temperature $T_{i}$ : when the temperatures are all equal to $T$ then $\tilde{L}_{S}=\frac{1}{T} L_{S}$. Computation yields,

$$
\tilde{L}_{S} H=\sum_{i=1}^{N} \gamma_{i}\left(1-\frac{p_{i}^{2}}{T_{i}}\right),
$$

and thus the average entropy flux due to the coupling to the heat baths in the measure $\mu$ is,

$$
\sigma_{\text {ext }}(\mu)=\sum_{i=1}^{N} \gamma_{i}\left\langle\left(1-\frac{p_{i}^{2}}{T_{i}}\right)\right\rangle_{\mu} .
$$

The Gibbs (or Shannon) entropy for any measure $\mu$ is,

$$
S(\mu)=-\int d x f \log f
$$

We define the entropy production in the chain as

$$
\left.s(\mu) \equiv \frac{d}{d t} S\left(\mu_{t}\right)\right|_{\mu_{t}=\mu}-\sigma_{e x t}(\mu) .
$$

We show now that with this definition, the entropy production is always positive. Note that,

$$
\frac{d}{d t} S\left(\mu_{t}\right)=-\int d x f_{t} L \log f_{t}
$$

As $L=L_{S}+L_{A}$ and $L_{A}$ is a first-order differential operator such that $L_{A}^{\dagger}=-L_{A}$, we see that

$$
\frac{d}{d t} S\left(\mu_{t}\right)=-\int d x f_{t}\left(L_{S}+L_{A}\right) \log f_{t}=-\int d x f_{t} L_{S} \log f_{t}
$$


Comparing the density $f_{t}$ with the reference measure $\rho_{\underline{\beta}}$ (see (17))

$$
\rho_{\underline{\beta}}=\frac{1}{Z_{\underline{\beta}}} \exp \left(-\sum_{i=1}^{N} \beta_{i} h_{i}\right)
$$

where $\beta_{i}=T_{i}^{-1}$ for every $i$ such that $\gamma_{i} \neq 0$ (other $\beta_{i}$ are arbitrary), we get,

$$
\begin{aligned}
\frac{d}{d t} S\left(\mu_{t}\right) & =-\int d x f_{t} L_{S} \log \frac{f_{t}}{\rho_{\underline{\beta}}}+\int d x f_{t} L_{S}\left(\sum_{i=1}^{N} \beta_{i} h_{i}\right) \\
& =-\int d x f_{t} L_{S} \log \frac{f_{t}}{\rho_{\underline{\beta}}}+\sum_{i=1}^{N} \gamma_{i} \int f_{t}\left(1-\frac{p_{i}^{2}}{T_{i}}\right) .
\end{aligned}
$$

We recall that $\Gamma(f, g)=2 \sum_{i=1}^{N} \gamma_{i} T_{i} \partial_{p_{i}} f \partial_{p_{i}} g$. For the first term, we note the identity,

$$
L_{S}(\log h)=h^{-1} L_{S} h-\frac{1}{2} h^{-2} \Gamma(h, h) .
$$

Combining ( $(\underline{55}),(\underline{59})$ and using the fact that $\left(L_{S}\right)^{\dagger} \rho_{\underline{\beta}}=0$ (because $\beta_{i}=T_{i}^{-1}$ for every $i$ such that $\gamma_{i} \neq 0$ ), with (57), this yields

$$
s(\mu)=\frac{1}{2} \int d x \rho_{\underline{\beta}}\left(\frac{\rho_{\underline{\beta}}}{f}\right) \Gamma\left(\frac{f}{\rho_{\underline{\beta}}}, \frac{f}{\rho_{\underline{\beta}}}\right)=2 \int d x \rho_{\underline{\beta}} \Gamma\left(\sqrt{\frac{f}{\rho_{\underline{\beta}}}}, \sqrt{\frac{f}{\rho_{\underline{\beta}}}}\right),
$$

with $f$ the density of the measure $\mu$. Since $\Gamma(f, f) \geq 0$, it is easy to see that $s(\mu) \geq 0$ and that the infimum is reached when $f=\rho_{\underline{\beta}}$. As already observed in [27], it is also obvious that, as $\Gamma$ only involves derivatives with respect to the variables $\underline{p}$, one can add any function of $\underline{q}$ in the exponential defining $\rho_{\beta}$. Therefore, even in the case of an equilibrium dynamics, when $T_{i}=T, \forall i$, such that $\gamma_{i} \neq 0$ in $L_{S}$, the minimum entropy production principle does not single out the equilibrium measure $\rho_{\beta}$. This comes from (58) which expresses the fact that the Gibbs entropy is invariant under the Hamiltonian evolution. Entropy is produced solely by the action of the thermostats which act only on the $\underline{p}$ variables.

As observed in [28], the large deviation functional $I$ provides a natural variational characterization of the stationary measure, which as we will see below, generalizes the minimum production entropy principle. As far as the variational principle is concerned, the key observation is that $I(\mu) \geq 0$ and $I(\mu)=0$ if and only if $\mu$ is a stationary measure for the process associated to the generator $L$ (Theorem 4.2.39 of [10]). We apply now Proposition 2 to the dynamics (34) which satisfies a generalized detailed balance (27) wrt the reference measure $\rho=\rho_{\underline{\beta}}$ (58) and $\sigma_{\underline{\beta}, \underline{\theta}}=\sum_{i=1}^{N-1}\left(\beta_{i}-\beta_{i+1}+\theta_{i}\right) j_{i}$. Thus (41) reads

$$
I(\mu)=\frac{1}{4} s(\mu)+K(\mu)-\frac{1}{2}\left\langle\sigma_{\underline{\beta}, \underline{\theta}}\right\rangle_{\mu},
$$

where we have used the following identity obtained by integration by parts from (60)

$$
\frac{1}{4} s(\mu)=-\left\langle f^{\frac{1}{2}} L_{S} f^{\frac{1}{2}}\right\rangle_{\rho_{\underline{\beta}}},
$$


for the reference measure $\rho_{\underline{\beta}}$. The first term in (61) is identified with the entropy production $s$ of the measure $\mu$, and as we have seen in the previous section, the second one $K$ records the coupling between the positions and momenta. The last term in (61) is the time-reversal breaking term given by combination of the microscopic currents. For equilibrium dynamics, by definition, the term $\sigma$ is absent, and the presence of $K$ ensures that the minimization of the sum of the first two terms in (61) yields the stationary measure univocally. Indeed, as we have explained above, minimizing entropy production alone is not sufficient to determine the equilibrium distribution. The example (52) of a single harmonic oscillator coupled to a heat bath is interesting in that respect and we come back briefly to it. We have already computed $K(\mu)$ in (53) and compute now the entropy production $s(\mu)$. We consider the reference measure,

$$
\rho(p, q)=\frac{1}{Z} \exp \left(-\frac{1}{T}\left(\frac{p^{2}}{2}+\omega^{2} \frac{q^{2}}{2}\right)\right),
$$

and a Gaussian measure

$$
\mu(q, p)=\frac{1}{\left(2 \pi b \omega^{2}\right)^{\frac{1}{2}}(2 \pi a)^{\frac{1}{2}}} \exp \left(-\left(a \frac{p^{2}}{2}+b \omega^{2} \frac{q^{2}}{2}\right)\right) .
$$

Then $s(\mu)=a^{-1} \gamma T(a-\beta)^{2}$. Combining this with the expression of $K(\mu)$ (53), we get,

$$
I(\mu)=\frac{1}{4 a} \gamma T(a-\beta)^{2}+\frac{1}{4 \gamma T}\left(\frac{b-a}{a}\right)^{2} \frac{\omega^{2}}{b} .
$$

In this simple example, we see that the role of $K$ is to ensure the equipartion of energy by the action of the Hamiltonian dynamics, the role of the entropy production is to fix the temperature of the equilibrium distribution.

\subsection{Positivity of the energy current in the asymmetric chain.}

We come back to the asymmetric chain defined in (32) and use the previous results on the representation of the Donsker-Varadhan functional to prove that when the parameter $\tau$ is different from zero then in the stationary state $\hat{\rho}, \tau\langle J\rangle_{\hat{\rho}}>0$. We note that the hypoellipticity of the process defined by the asymmetric chain may be shown by checking the Hörmander condition on the generator as in [34]. For the asymmetric periodic chain, no coupling between nearest-neighbour is even required because the noise acts on every particle. Hypoellipticity of the process implies the smoothness of the probability transition and thus that the stationary state, whenever it exists, is described by a smooth density. Irreducibility properties of the process may be checked by a control argument and Stroock-Varadhan support theorem [38] as explained in [9, 34. Hypoellipticity and irreducibility imply together that there is at most one stationary measure. The existence of a (unique) stationary measure follows from those two properties and the existence of a Lyapunov function as in (102) in the appendix (see for instance Theorem 8.7 of [33]).

Proposition 4 Let $U$ and $V$ be potentials satisfying the hypothesis of proposition 1 and such that $U^{\prime \prime}$ is not uniformly equal to 0 . Let $\hat{\rho}$ be the unique stationary state for the dynamics (25), then $\tau\langle J\rangle_{\hat{\rho}} \geq 0$. If $\tau \neq 0$, then $\tau\langle J\rangle_{\hat{\rho}}>0$. 
Proof. Take as a reference measure $\rho_{\beta}=Z^{-1} \exp (-\beta H)$, it was noted in (33) that the dynamics satisfies a generalized detailed balance relation with respect to $\rho_{\beta}$ and $\sigma$, with $\sigma=\frac{\tau N}{T^{2}} J, T=\beta^{-1}$. Write the formula (41) of Proposition 2 under the form (61),

$$
I(\mu)=\frac{1}{4} s(\mu)+K(\mu)-\frac{1}{2}\langle\sigma\rangle_{\mu},
$$

for a smooth measure $\mu$. As $\hat{\rho}$ is a smooth stationary measure, then $I(\hat{\rho})=0$. Since $s(\hat{\rho})$ and $K(\hat{\rho})$ are positive, one concludes that $\tau\langle J\rangle_{\hat{\rho}} \geq 0$, using (65). To show that if $\tau \neq 0,\langle J\rangle_{\hat{\rho}}>0$, we proceed by contradiction. Assume then that $\tau \neq 0$ and $\langle J\rangle_{\hat{\rho}}=0$, then simultaneously $s(\hat{\rho})=0$ and $K(\hat{\rho})=0$, since the two are always non-negative. Writing $\hat{\rho}=\rho_{\beta} \exp (-\varphi)$, the first condition implies by construction of $s(\hat{\rho})$, (see (60) ) that,

$$
\partial_{p_{i}} \varphi=0, \forall i \text {. }
$$

On the other hand, $K(\hat{\rho})=0$ implies that the solution $\bar{W}$ in (49) is such that $\partial_{p_{i}} \bar{W}=0, \forall i$. Therefore, using (48), $\varphi$ must be such that,

$$
L_{A}(\beta H+\varphi)=0
$$

Since $L_{A}=L_{H}+L_{\tau}$ and combining (25) with (66), $\varphi$ satisfies

$$
\sum_{i=1}^{N} p_{i} \partial_{q_{i}} \varphi=\frac{\tau}{2 T} \sum_{i=1}^{N} p_{i} \Psi_{i}
$$

with $\Psi_{i}=U^{\prime}\left(q_{i-1}-q_{i}\right)+U^{\prime}\left(q_{i}-q_{i+1}\right)$. As this equation holds for any vector $\underline{p}$ and because by (66),$\varphi$ does not depend on $\underline{p}$, it means that

$$
\partial_{q_{i}} \varphi=\frac{\tau}{2 T} \Psi_{i}, \forall i
$$

But since $\partial_{q_{i}} \Psi_{i+1}=U^{\prime \prime}\left(q_{i}-q_{i+1}\right)$ and $\partial_{q_{i+1}} \Psi_{i}=-U^{\prime \prime}\left(q_{i}-q_{i+1}\right)$, there cannot be any solution to (69) , unless $U^{\prime \prime}(q)=0$ for all $q$. Therefore, we conclude that $\langle J\rangle_{\hat{\rho}} \neq 0$ and thus $\tau\langle J\rangle_{\hat{\rho}}>0$, because $\tau\langle J\rangle_{\hat{\rho}} \geq 0$.

\subsection{The Gallavotti-Cohen symmetry}

An interesting aspect of (41) in Proposition 2,

$$
I(\mu)=-\left\langle f^{\frac{1}{2}} L_{S} f^{\frac{1}{2}}\right\rangle_{\rho}+K(\mu)-\frac{1}{2}\langle\sigma\rangle_{\mu}
$$

is that when the triple $(L, \rho, \sigma)$ satisfies a generalized detailed balance relation, the three terms are either odd or even under the reversal of momenta $\Pi$. The fact that $K(\Pi \mu)=K(\mu)$ was established in Proposition 3. In the previous section, we have identified the first term in (70) to the entropy production $s(\mu)$. It is also invariant under time-reversal (i.e $s(\Pi \mu)=s(\mu)$ ) when $\Pi \rho=\rho$ because by construction $\Pi L_{S} \Pi=L_{S}$. And, since $\sigma$ is odd under time reversal $(\Pi \sigma=-\sigma)$, one may therefore write,

$$
I(\Pi \mu)=-\left\langle f^{\frac{1}{2}} L_{S} f^{\frac{1}{2}}\right\rangle_{\rho}+K(\mu)+\frac{1}{2}\langle\sigma\rangle_{\mu}=I(\mu)+\langle\sigma\rangle_{\mu} .
$$

Heuristically, (71) implies the Gallavotti-Cohen symmetry for the large deviations of the function $\sigma$ integrated over time

$$
\lim _{t \rightarrow \infty}-\frac{1}{t} \log P\left[\frac{1}{t} \int_{0}^{t} \sigma(s) d s=\hat{\sigma}\right]=\hat{I}(\hat{\sigma}),
$$


with

$$
\hat{I}(\hat{\sigma})=\inf _{\mu}\left\{I(\mu) \mid\langle\sigma\rangle_{\mu}=\hat{\sigma}\right\}
$$

Since $\Pi \sigma=-\sigma$,

$$
\begin{aligned}
\hat{I}(-\hat{\sigma}) & =\inf _{\mu}\left\{I(\mu) \mid\langle\sigma\rangle_{\mu}=-\hat{\sigma}\right\}=\inf _{\mu}\left\{I(\mu) \mid\langle\Pi \sigma\rangle_{\mu}=\hat{\sigma}\right\} \\
& =\inf _{\mu}\left\{I(\mu) \mid\langle\sigma\rangle_{\Pi \mu}=\hat{\sigma}\right\}=\inf _{\mu}\left\{I(\Pi \mu) \mid\langle\sigma\rangle_{\mu}=\hat{\sigma}\right\} .
\end{aligned}
$$

Thus one gets from (71)

$$
\hat{I}(\hat{\sigma})-\hat{I}(-\hat{\sigma})=-\hat{\sigma} .
$$

This is the celebrated Gallavotti-Cohen symmetry relation [18, 19, 16, 21, 22, 23, 26]. For a chain of oscillators in contact with two heat baths, the Gallavotti-Cohen symmetry has been rigorously justified in [14, 35].

\section{Large deviations of the current}

Using the Donsker-Varadhan variational principle (41), we are going derive explicit expressions for the large deviation function in the case of harmonic interaction when the system size diverges. We consider the asymmetric dynamics (32) on a periodic chain of length $N$ where each oscillator is coupled to a heat bath at temperature $T$

$$
\begin{aligned}
& d q_{i}=p_{i} d t \\
& d p_{i}=-\gamma p_{i} d t-\frac{\partial H}{\partial q_{i}} d t+\frac{\tau \omega^{2}}{2 T}\left(q_{i+1}-q_{i-1}\right) d t+\sqrt{2 \gamma T} d w_{i},
\end{aligned}
$$

with harmonic potential

$$
H(\underline{q}, \underline{p})=\sum_{i=1}^{N} \frac{p_{i}^{2}}{2}+\frac{\omega_{0}^{2}}{2} q_{i}^{2}+\frac{\omega^{2}}{2}\left(q_{i}-q_{i+1}\right)^{2},
$$

and a pinning $\omega_{0}>0$.

We will study the large deviations of the spatial average of the total current (7)

$$
J(\underline{q}, \underline{p})=\frac{\omega^{2}}{2 N} \sum_{i=1}^{N}\left(q_{i+1}-q_{i}\right)\left(p_{i}+p_{i+1}\right) .
$$

The large deviation principle (Proposition 1) implies that for $\lambda$ and $\tau$ small enough

$$
\mathcal{F}_{\lambda, N}^{\tau}=\lim _{t \rightarrow \infty} \frac{1}{t} \log P\left(\exp \left(N \lambda \int_{0}^{t} d s J(\underline{q}(s), \underline{p}(s))\right)\right),
$$

where

$$
\mathcal{F}_{\lambda, N}^{\tau}=\sup _{\mu}\left\{\mathcal{F}_{\lambda, N}^{\tau}(\mu)\right\} \quad \text { with } \quad \mathcal{F}_{\lambda, N}^{\tau}(\mu)=N \lambda\langle J\rangle_{\mu}-I^{\tau}(\mu),
$$

where the upper-script $\tau$ emphasizes the dependency on the asymmetry. We recall from Proposition 2 that $I^{\tau}(\mu)$ can be written,

$$
I^{\tau}(\mu)=\frac{1}{4} s(\mu)+K^{\tau}(\mu)-\frac{\tau N}{2 T^{2}}\langle J\rangle_{\mu},
$$

where $s(\mu)$ denotes the entropy production (62).

For the harmonic chain (74), Gaussian computations lead to the following asymptotics for $\mathcal{F}_{\lambda, N}^{\tau}$ 


\section{Proposition 5}

(i) For $\tau=0$ and fixed $N$, one has for $\lambda$ small

$$
\mathcal{F}_{\lambda, N}^{0}=\sum_{k=1}^{N} \frac{T^{2} \omega^{4}}{\gamma \omega_{k}^{2}} \sin \left(2 \pi \frac{k}{N}\right)^{2} \lambda^{2}+\left(\frac{T^{4} \omega^{8}}{\gamma \omega_{k}^{6}}+5 \frac{T^{4} \omega^{8}}{\gamma^{3} \omega_{k}^{4}}\right) \sin \left(2 \pi \frac{k}{N}\right)^{4} \lambda^{4}+O\left(\lambda^{6}\right),
$$

with $\omega_{k}^{2}=\omega_{0}^{2}+4 \omega^{2} \sin \left(2 \pi \frac{k}{N}\right)^{2}$.

(ii) Fix $\omega_{0}>0$ and two parameters $\lambda^{\prime}, \tau^{\prime} \in \mathbf{R}$. For large $N$, with the scaling $\lambda=\lambda^{\prime} / N$ and $\tau=\tau^{\prime} / N$, one gets

$$
\lim _{N \rightarrow \infty} N \mathcal{F}_{\lambda, N}^{\tau}=\kappa\left(\lambda^{\prime} \tau^{\prime}+\left(\lambda^{\prime}\right)^{2} T^{2}\right)
$$

where $\kappa$ is the conductivity of the model

$$
\kappa=\frac{1}{\gamma} \int_{-\frac{1}{2}}^{\frac{1}{2}} \frac{\omega^{4} \sin ^{2}(2 \pi x)}{\omega_{0}^{2}+4 \omega^{2} \sin ^{2}(2 \pi x)} d x
$$

Before deriving Proposition 5, we first comment on the results. Taking the Legendre transform of (81) shows that for a weak drift $\tau=\frac{\tau^{\prime}}{N}$, the large deviations of the currrent are given by

$$
\lim _{t \rightarrow \infty}-\frac{1}{t} \log P\left(\int_{0}^{t} d s J(\underline{q}(s), \underline{p}(s)) \simeq \frac{j}{N}\right)=\hat{I}_{N}(j)
$$

where $\hat{I}_{N}$ scales, for large $N$, like

$$
\hat{I}_{N}(j)=\frac{1}{N} \frac{\left(j-\kappa \tau^{\prime}\right)^{2}}{4 \kappa T^{2}}+O\left(\frac{1}{N^{2}}\right) .
$$

In the stationary regime, the mean current has been already computed in terms of the conductivity (82) (see [24])

$$
\lim _{N \rightarrow \infty} \lim _{t \rightarrow \infty} N\langle J(\underline{q}(t), \underline{p}(t))\rangle=\kappa \tau^{\prime} .
$$

The expression (83) is new in the framework of thermostated Hamiltonian systems: it relates the linear response theory [24] to the large deviations and shows that the atypical behavior can still be described by the conductivity $\kappa$. The reason is that the local equilibrium is preserved for small shifts of the current (remark that the fourth order term in (80) corresponds to corrections to local equilibrium). For weak current deviations, (83) has a Gaussian structure similar to the one obtained in lattice gases with a weak asymmetry [5, 3]. The variance in (83) (given by $2 \kappa T^{2}$ ) is related to the response coefficient $\kappa$ according to the usual Einstein fluctuation-dissipation. In the derivation of (80), we will see that the corrections due to $K^{\tau=0}$ arise only at the order $\lambda^{4}$. The functional is only quadratic asymptotically in $N$. The Gaussian structure will not remain for non vanishing drifts $\tau$ or larger current deviations. A similar behavior can be observed in lattice gases like the weakly/totally asymmetric exclusion processes [5]. 
As the system is periodic and the functional $\mathcal{F}_{\lambda, N}^{\tau}$ is convex, one can look for a maximizer of the variational principle (78) among the translation-invariant measures. The current $J$ in (7) is quadratic and the evolution equations (74) linear, thus the minimum in (78) will be reached for Gaussian measures (to see this, one can check that the maximizer of (178) is obtained in terms of the principle eigenvector of the operator $L-\lambda J$ and the principle eigenvector of its adjoint). Thus it remains to optimize the variational principle (78) over Gaussian translation-invariant measures. We state first Gaussian estimates before deriving (i) and (ii).

\section{Gaussian estimates:}

For periodic systems, the Gaussian computations are easier in Fourier coordinates. We set

$$
P_{k}=\frac{1}{\sqrt{N}} \sum_{j=1}^{N} e^{i \frac{2 \pi}{N} k j} p_{j}, \quad Q_{k}=\frac{1}{\sqrt{N}} \sum_{j=1}^{N} e^{i \frac{2 \pi}{N} k j} q_{j}
$$

For notational simplicity, we choose an odd length $N=2 n+1$ and the Fourier modes $k \in\{-n, n\}$. This choice leads to symmetric relations: $\bar{P}_{k}=P_{-k}$ and thus, $\left|P_{k}\right|^{2}=P_{-k} P_{k}$ and similarly for the variables $Q_{k}$.

We will first provide explicit expressions of $I^{\tau}(\mu)$ for a general Gaussian measure $\mu$. Given the reference measure $\rho_{0}$

$$
\rho_{0}=\exp \left(-\frac{1}{2 T} \sum_{k}\left|P_{k}\right|^{2}+\omega_{k}^{2}\left|Q_{k}\right|^{2}\right)
$$

the most general form for a Gaussian translation-invariant measure in the variables $(\underline{P}, \underline{Q})$ may be written $\mu=Z^{-1} \exp \left(-\frac{1}{T} \Psi\right) \rho_{0}$, with

$$
\Psi=\sum_{k=-n}^{n}\left(\mathrm{i} a_{k} P_{k} Q_{-k}+b_{k}\left[\left|P_{k}\right|^{2}+\omega_{k}^{2}\left|Q_{k}\right|^{2}\right]+c_{k}\left[\left|P_{k}\right|^{2}-\omega_{k}^{2}\left|Q_{k}\right|^{2}\right]\right)
$$

with $a_{k}=\Re\left(a_{k}\right)+\mathrm{i} \Im\left(a_{k}\right)=-\bar{a}_{-k} \in \mathbf{C}$ and $b_{k}=b_{-k} \in \mathbf{R}, c_{k}=c_{-k} \in \mathbf{R}$. The expectation under $\mu$ will be denoted $<\cdot>_{\mu}$, and for each mode $k \in\{-n, n\}$

$$
\begin{aligned}
& \left\langle\left|P_{k}\right|^{2}\right\rangle_{\mu}=\delta_{k}\left(1+2\left(b_{k}-c_{k}\right)\right) \omega_{k}^{2} T, \quad\left\langle\left|Q_{k}\right|^{2}\right\rangle_{\mu}=\delta_{k}\left(1+2\left(b_{k}+c_{k}\right)\right) T \\
& \mathrm{i}\left\langle P_{k} Q_{-k}-P_{-k} Q_{k}\right\rangle_{\mu}=-2 \Re\left(a_{k}\right) \delta_{k} T, \quad\left\langle P_{k} Q_{-k}+P_{-k} Q_{k}\right\rangle_{\mu}=-2 \Im\left(a_{k}\right) \delta_{k} T
\end{aligned}
$$

with $\delta_{k}^{-1}=\left(1+2 b_{k}\right)^{2} \omega_{k}^{2}-4 c_{k}^{2} \omega_{k}^{2}-\left|a_{k}\right|^{2}$.

Using now Proposition 3, we are going to compute $K^{\tau}(\mu)$ for the dynamics and $\mu$ as above. In Fourier coordinates the antisymmetric part of the generator $L_{A}=L_{H}+L_{\tau}(? ?)$ is given by

$$
L_{H}=\sum_{k=-n}^{n} P_{k} \frac{\partial}{\partial Q_{k}}-\omega_{k}^{2} Q_{k} \frac{\partial}{\partial P_{k}}
$$

with $\omega_{k}^{2}=\omega_{0}^{2}+4 \omega^{2} \sin \left(2 \pi \frac{k}{N}\right)^{2}$ and

$$
L_{\tau}=-\mathrm{i} \frac{\tau \omega^{2}}{T} \sum_{k=-n}^{n} \sin \left(\frac{2 \pi k}{N}\right) Q_{k} \frac{\partial}{\partial P_{k}} .
$$


We first show that if $\Im\left(a_{k}\right) \neq 0$ for some $k$, then $K^{\tau}(\mu)=+\infty$. Remark that $L_{A}\left|Q_{k}\right|^{2}=P_{k} Q_{-k}+P_{-k} Q_{k}$ and that according to (87), $\left\langle L_{A}\left|Q_{k}\right|^{2}\right\rangle_{\mu}=0$ if and only if $\Im\left(a_{k}\right)=0$. From the definition of $K^{\tau}(\mu)(42)$, if $\Im\left(a_{k}\right) \neq 0$ for some $k$, it is possible to choose a sequence of real numbers $\zeta_{n}$ such that for $W_{n}=\zeta_{n}\left|Q_{k}\right|^{2}$

$$
\lim _{n \rightarrow \infty}\left(\frac{1}{8}\left\langle\Gamma\left(W_{n}, W_{n}\right)\right\rangle_{\mu}+\frac{1}{2}\left\langle L_{A} W_{n}\right\rangle_{\mu}\right)=\lim _{n \rightarrow \infty} \frac{1}{2} \zeta_{n}\left\langle P_{k} Q_{-k}+P_{-k} Q_{k}\right\rangle_{\mu}=-\infty,
$$

and thus $K^{\tau}(\mu)=+\infty$. So from now on, we assume that $a_{k}$ is real and that $a_{k}=-a_{-k}$. In that case a solution of (48) is

$$
\bar{W}=\sum_{k=-n}^{n} \sin \left(2 \pi \frac{k}{N}\right) \frac{\mathrm{i} \tau \omega^{2}}{2 \gamma T^{2}}\left(P_{k} Q_{-k}-P_{-k} Q_{k}\right)+\frac{c_{k} \omega_{k}^{2}}{\left(\frac{1}{2}+b_{k}+c_{k}\right) \gamma T}\left(P_{k} Q_{-k}+P_{-k} Q_{k}\right) .
$$

By Proposition 3, one gets,

$$
K^{\tau}(\mu)=\frac{1}{\gamma} \sum_{k=-n}^{n} \delta_{k}\left(1+2\left(b_{k}+c_{k}\right)\right)\left(\frac{\tau^{2} \omega^{4}}{4 T^{2}} \sin \left(2 \pi \frac{k}{N}\right)^{2}+\frac{4 c_{k}^{2} \omega_{k}^{4}}{\left(1+2\left(b_{k}+c_{k}\right)\right)^{2}}\right),
$$

with $\omega_{k}^{2}=\omega_{0}^{2}+4 \omega^{2} \sin \left(2 \pi \frac{k}{N}\right)^{2}$ and $\delta_{k}^{-1}=\left(1+2 b_{k}\right)^{2} \omega_{k}^{2}-4 c_{k}^{2} \omega_{k}^{2}-a_{k}^{2}$.

Note that $K^{\tau}(\mu)$ is made of two parts: an equilibrium one (independent of the forcing $\tau$ ) and of a non-equilibrium one.

The entropy production (60) is given by

$$
\begin{aligned}
s(\mu) & =\frac{\gamma}{T} \sum_{k=-n}^{n}\left\langle\frac{\partial \Psi}{\partial P_{k}} \frac{\partial \Psi}{\partial P_{-k}}\right\rangle_{\mu} \\
& =\frac{\gamma}{T} \sum_{k} 2 \mathrm{i} a_{k}\left(b_{k}+c_{k}\right)\left\langle P_{k} Q_{-k}-P_{-k} Q_{k}\right\rangle_{\mu}+a_{k}^{2}\left\langle\left|Q_{k}\right|^{2}\right\rangle_{\mu}+4\left(b_{k}+c_{k}\right)^{2}\left\langle\left|P_{k}\right|^{2}\right\rangle_{\mu} .
\end{aligned}
$$

For $a_{k}$ real, one gets from (87)

$$
s(\mu)=\gamma \sum_{k=-n}^{n} \delta_{k}\left[a_{k}^{2}\left(1-2\left(b_{k}+c_{k}\right)\right)+4\left(b_{k}+c_{k}\right)^{2} \omega_{k}^{2}\left(1+2\left(b_{k}-c_{k}\right)\right)\right] .
$$

Finally, the current can be represented in Fourier coordinates

$$
J=-\mathrm{i} \frac{\omega^{2}}{2 N} \sum_{k=-n}^{n} \sin \left(2 \pi \frac{k}{N}\right)\left(P_{k} Q_{-k}-P_{-k} Q_{k}\right)
$$

and from (87), its expectation is

$$
<>_{\mu}=\frac{\omega^{2} T}{N} \sum_{k=-n}^{n} \sin \left(2 \pi \frac{k}{N}\right) a_{k} \delta_{k} .
$$

\section{Proof of (i) :}

The functional (78) reads for $\tau=0$

$$
\mathcal{F}_{\lambda, N}^{\tau}(\mu)=-\frac{1}{4} s(\mu)-K^{0}(\mu)+N \lambda\langle J\rangle_{\mu} .
$$


Restricting to translation invariant Gaussian measures (86), the previous Gaussian computation lead to

$$
\begin{aligned}
\mathcal{F}_{\lambda, N}(\mu)= & \sum_{k=-n}^{n} \delta_{k}\left[\lambda T \omega^{2} \sin \left(2 \pi \frac{k}{N}\right) a_{k}-\frac{1}{\gamma} \frac{4 c_{k}^{2} \omega_{k}^{4}}{\left(1+2\left(b_{k}+c_{k}\right)\right)}\right. \\
& \left.-\frac{\gamma}{4}\left(a_{k}^{2}\left(1-2\left(b_{k}+c_{k}\right)\right)+4\left(b_{k}+c_{k}\right)^{2} \omega_{k}^{2}\left(1+2\left(b_{k}-c_{k}\right)\right)\right)\right]
\end{aligned}
$$

Optimizing over each Fourier mode leads at the order $\lambda^{2}$

$$
\begin{aligned}
& a_{k}=\frac{2 T \omega^{2}}{\gamma} \sin \left(2 \pi \frac{k}{N}\right) \lambda+O\left(\lambda^{3}\right), \quad b_{k}=-\left(\frac{T^{2} \omega^{4}}{2 \omega_{k}^{4}}+\frac{T^{2} \omega^{4}}{\gamma^{2} \omega_{k}^{2}}\right) \sin \left(2 \pi \frac{k}{N}\right)^{2} \lambda^{2} \\
& c_{k}=\frac{T^{2} \omega^{4}}{2 \omega_{k}^{4}} \sin \left(2 \pi \frac{k}{N}\right)^{2} \lambda^{2} .
\end{aligned}
$$

The previous computations imply that at the order $\lambda$ the solution of the variational principle (78) is given by

$$
\mu(\underline{q}, \underline{p})=\frac{1}{Z} \exp \left(\frac{2 \lambda N}{\gamma} J(\underline{q}, \underline{p})\right) \rho_{0}(\underline{q}, \underline{p}) .
$$

Finally, asymptotics (80) follow from (25).

\section{Proof of (ii) :}

We observe that (78) may be rewritten

$$
\sup _{\mu} \mathcal{F}_{\lambda, N}^{\tau}(\mu)=\sup _{\mu}\left[-I^{\tau+2 \lambda T^{2}}(\mu)-K^{\tau}(\mu)+K^{\tau+2 \lambda T^{2}}(\mu)\right] .
$$

The point of writing the variational problem under this form is that minimizing only $I^{\tau+2 \lambda T^{2}}$ is easy. The minimizer is the stationary measure of the asymmetric periodic chain with non-equilibrium parameter $\tau$ replaced by $\tau+2 \lambda T^{2}$. It was derived in [24],

$$
\rho_{\tau+2 \lambda T^{2}}(\underline{q}, \underline{p})=\frac{1}{Z} \exp \left(-\frac{1}{T} H(\underline{q}, \underline{p})+\frac{N}{\gamma T^{2}}\left(\tau+2 \lambda T^{2}\right) J(\underline{q}, \underline{p})\right) .
$$

For small $\lambda$ and $\tau$, we will see that $\rho_{\tau+2 \lambda T^{2}}$ is a good approximation of the minimizer of the variational principle $\mathcal{F}_{\lambda, N}^{\tau}$ at the lowest order (see also (96)).

$I^{\tau+2 \lambda T^{2}}$ is a convex function of the parameters $\left(a_{k}, b_{k}, c_{k}\right)$. Since $\omega_{0}^{2}>0$, the contributions of each modes are independent with an hessian uniformly bounded from below in a neighborhood of the minimizer $\rho_{\tau+2 \lambda T^{2}}$. Any measure of the form $\mu=Z^{-1} \exp \left(-\frac{1}{T} \Psi\right) \rho_{\tau+2 \lambda T^{2}}$, with $\Psi$ as in (86) , has a contribution

$$
I^{\tau+2 \lambda T^{2}}(\mu) \geq C \sum_{k=-n}^{n}\left\{a_{k}^{2}+b_{k}^{2}+c_{k}^{2}\right\}
$$

for some constant $C>0$ independent of $N$ and $\left(a_{k}, b_{k}, c_{k}\right)$ close to 0 . 
From the exact expression (91), we first evaluate the difference in (97) for a general Gaussian measure (86)

$$
\begin{aligned}
K^{\tau+2 \lambda T^{2}}(\mu)-K^{\tau}(\mu) & =\left(\left(\tau+2 \lambda T^{2}\right)^{2}-\tau^{2}\right) \frac{1}{\gamma} \sum_{k} \delta_{k}\left(1+2\left(b_{k}+c_{k}\right)\right) \frac{\omega^{4}}{4 T^{2}} \sin \left(2 \pi \frac{k}{N}\right)^{2} \\
& =\left(\lambda \tau+\lambda^{2} T^{2}\right) \frac{1}{\gamma} \sum_{k} \delta_{k}\left(1+2\left(b_{k}+c_{k}\right)\right) \omega^{4} \sin \left(2 \pi \frac{k}{N}\right)^{2} \cdot \quad
\end{aligned}
$$

For large $N$, with the scaling $\lambda=\lambda^{\prime} / N$ and $\tau=\tau^{\prime} / N$, the contribution of the $k^{t h}$-Fourier mode is at most of order $\frac{1}{N^{2}} \sin \left(2 \pi \frac{k}{N}\right)^{2}$. From (99), the corrections $\left(a_{k}, b_{k}, c_{k}\right)$ have to remain much smaller than $\frac{1}{N} \sin \left(2 \pi \frac{k}{N}\right)$, otherwise the contribution of $I^{\tau+2 \lambda T^{2}}$ would be too large as compared to $K^{\tau+2 \lambda T^{2}}-K^{\tau}$. This shows that at the lowest order $\rho_{\tau+2 \lambda T^{2}}$ approximates correctly the minimizer of $\mathcal{F}_{\lambda, N}^{\tau}$. Plugging the expression (98) of $\rho_{\tau+2 \lambda T^{2}}$ in (97) and then in (100) gives at the lowest order in $1 / N$

$$
\mathcal{F}_{\lambda, N}^{\tau}\left(\rho_{\tau+2 \lambda T^{2}}\right)=\frac{\left(\lambda^{\prime} \tau^{\prime}+\left(\lambda^{\prime}\right)^{2} T^{2}\right)}{N^{2}} \frac{\omega^{4}}{\gamma} \sum_{k} \frac{1}{\omega_{k}^{2}} \sin \left(2 \pi \frac{k}{N}\right)^{2}+o\left(\frac{1}{N}\right)
$$

For large $N$, the last sum may be approximated by an integral

$$
\mathcal{F}_{\lambda, N}^{\tau}\left(\rho_{\tau+2 \lambda T^{2}}\right)=\frac{\kappa}{N}\left(\lambda^{\prime} \tau^{\prime}+\left(\lambda^{\prime}\right)^{2} T^{2}\right)+o\left(\frac{1}{N}\right)
$$

where $\kappa$ is the conductivity of the model (82).

Remark. Note that a direct computation of the largest eigenvalue of the operator $L-\lambda J$ would have led also to $\mathcal{F}_{\lambda, N}^{\tau}$.

\section{Conclusion}

We have analyzed the large deviations for thermostated lattices of Hamiltonian oscillators by using the Donsker-Varadhan large deviation theory [11, 12. After deriving the large deviation principle for a large class of models (Proposition 1), we analyzed the Donsker-Varadhan functional (41) and showed in section 3.2 that it provides a variational characterization of the stationary measure which generalizes the minimum entropy production principle.

The current large deviation function has been computed exactly (Proposition 5) for thermostated lattices of harmonic oscillators on a ring. The central issue in this computation is the evaluation of the "dynamical activity" $K$ of the stationary measures of systems out of equilibrium. In this paper, we used the fact that the explicit form of this measure is known in the model treated. It would be interesting to generalize these computations to Gaussian self-consistent reservoirs for which the temperatures of the heat baths vary spatially. Except for the lack of translation invariance, this model is very similar to the one considered here. A very challenging issue would be to investigate the current large deviations of the chain of non-harmonic oscillators. Our analysis of the Donsker-Varadhan functional applies 
to this type of dynamics, but to derive quantitative estimates of the large deviations of the current, some type of approximation seems necessary. In the approximation [24, 25, 1, 17, 37], the action of the nonlinearity of the dynamics is essentially reduced to the action of Gaussian stochastic heat baths, thus one should be able to proceed in similar fashion as in the model studied in the previous section.

\section{Appendix}

In this appendix, we prove Proposition 1 for the dynamics (34). The generator of this dynamics is given by $L=L_{S}+L_{H}+L_{\underline{\theta}}$, with

$$
\begin{aligned}
& L_{S}=\sum_{i=1}^{N}-\gamma_{i} p_{i} \frac{\partial}{\partial p_{i}}+\gamma_{i} T_{i} \frac{\partial^{2}}{\partial p_{i}^{2}}, \quad L_{H}=\sum_{i=1}^{N}-\frac{\partial H}{\partial q_{i}} \frac{\partial}{\partial p_{i}}+\frac{\partial H}{\partial p_{i}} \frac{\partial}{\partial q_{i}}, \\
& L_{\underline{\theta}}=-\sum_{i=1}^{N} \frac{1}{2} T_{i}\left(\theta_{i-1} U^{\prime}\left(q_{i-1}-q_{i}\right)+\theta_{i} U^{\prime}\left(q_{i}-q_{i+1}\right)\right) \frac{\partial}{\partial p_{i}}, \quad \text { with } \quad \theta_{0}=\theta_{N}=0,
\end{aligned}
$$

and open boundary conditions $\left(q_{0}=q_{N+1}=0\right)$. The case of periodic boundary conditions can be treated similarly.

The proof heavily relies on corollary 2.2 of the paper [39] where it is proven that the large deviation principle applies provided that one can find a function $\Psi \geq 1$ such that the function

$$
\Phi(\underline{p}, \underline{q})=-\frac{L \Psi}{\Psi}(\underline{p}, \underline{q})
$$

diverges at infinity. This can be understood as follows. From (102), we deduce that $\Psi$ is an eigenvector for the operator $L+\Phi$, thus the Feynman-Kac formula implies that for any time $t$ and initial data $(\underline{p}(0), \underline{q}(0))$

$$
\begin{aligned}
\Psi(\underline{p}(0), \underline{q}(0))=P & \left(\Psi(\underline{p}(t), \underline{q}(t)) \exp \left(\int_{0}^{t} d s \Phi(\underline{p}(s), \underline{q}(s))\right)\right) \\
& \geq P\left(\exp \left(\int_{0}^{t} d s \Phi(\underline{p}(s), \underline{q}(s))\right)\right)
\end{aligned}
$$

where we used that $\Psi \geq 1$ in the last inequality. This ensures the exponential integrability of $\int_{0}^{t} d s \Phi(\underline{p}(s), \underline{q}(s))$ uniformly in time. Since $\Phi$ diverges at infinity, we recover tightness properties and the existence of an invariant measure for the dynamics (see Theorem 3.1 in [39]).

Proof. Following [39], we introduce $\Psi(\underline{p}, \underline{q})=\exp (F(\underline{p}, \underline{q})-\inf F)$ with

$$
F=\frac{1}{2} \hat{H}(\underline{p}, \underline{q})+b \sum_{i=1}^{N} q_{i} p_{i}
$$

with $b$ a small constant and $\hat{H}$ given by (see (17))

$$
\hat{H}(\underline{p}, \underline{q})=\sum_{i=1}^{N} \frac{p_{i}^{2}}{2 T_{i}}+\frac{1}{T_{i}} V\left(q_{i}\right)+\frac{U\left(q_{i}-q_{i+1}\right)+U\left(q_{i-1}-q_{i}\right)}{2 T_{i}} .
$$


From (102), we get

$$
\Phi(\underline{p}, \underline{q})=-L F(\underline{p}, \underline{q})-\sum_{i=1}^{N} \gamma_{i} T_{i}\left(\partial_{p_{i}} F\right)^{2} .
$$

We compute each terms

$$
\begin{gathered}
L \hat{H}(\underline{p}, \underline{q})=\sum_{i=1}^{N} \gamma_{i}-\frac{\gamma_{i}}{T_{i}} p_{i}^{2}-\sum_{i=1}^{N-1}\left(\frac{1}{2 T_{i}}-\frac{1}{2 T_{i+1}}\right) U^{\prime}\left(q_{i}-q_{i+1}\right)\left(p_{i}+p_{i+1}\right) \\
-\sum_{i=1}^{N} \frac{1}{2}\left(\theta_{i} U^{\prime}\left(q_{i}-q_{i+1}\right)+\theta_{i-1} U^{\prime}\left(q_{i-1}-q_{i}\right)\right) p_{i}
\end{gathered}
$$

where $L_{H} \hat{H}$ was already computed in (20). One has also

$$
\begin{aligned}
& L q_{i} p_{i}=-\gamma_{i} p_{i} q_{i}+p_{i}^{2}-q_{i} \partial_{q_{i}} H(q)-\frac{T_{i}}{2}\left(\theta_{i} U^{\prime}\left(q_{i}-q_{i+1}\right)+\theta_{i-1} U^{\prime}\left(q_{i-1}-q_{i}\right)\right) q_{i} \\
& \left(\partial_{p_{i}} F\right)^{2}=\left(\frac{1}{2 T_{i}} p_{i}+b q_{i}\right)^{2} .
\end{aligned}
$$

Combining the previous results leads to

$$
\begin{aligned}
\Phi(\underline{p}, \underline{q})= & \sum_{i=1}^{N}\left(\frac{\gamma_{i}}{4 T_{i}}-b\right) p_{i}^{2}+b q_{i} \partial_{q_{i}} H(q)-b^{2} \gamma_{i} T_{i} q_{i}^{2}-\frac{\gamma_{i}}{2} \\
& +\frac{1}{2}\left(\theta_{i} U^{\prime}\left(q_{i}-q_{i+1}\right)+\theta_{i-1} U^{\prime}\left(q_{i-1}-q_{i}\right)\right)\left(b T_{i} q_{i}+\frac{p_{i}}{2}\right) \\
& +\frac{1}{4} \sum_{i=1}^{N-1}\left(\frac{1}{T_{i}}-\frac{1}{T_{i+1}}\right) U^{\prime}\left(q_{i+1}-q_{i}\right)\left(p_{i}+p_{i+1}\right)
\end{aligned}
$$

Since $U$ and $V$ are convex, one gets

$$
\sum_{i=1}^{N} q_{i} \partial_{q_{i}} H(q) \geq \sum_{i=1}^{N} V\left(q_{i}\right)+\frac{U\left(q_{i}-q_{i+1}\right)+U\left(q_{i+1}-q_{i}\right)}{2}-V(0)-U(0) .
$$

Combining this inequality and (106), we get

$$
\begin{aligned}
\Phi(\underline{p}, \underline{q}) \geq & \sum_{i=1}^{N}\left(\frac{\gamma_{i}}{4 \mathcal{T}_{+}}-b\right) p_{i}^{2}+b V\left(q_{i}\right)+b U\left(q_{i}-q_{i+1}\right)-b V(0)-b U(0)-\frac{\gamma_{i}}{2} \\
& -b^{2}\left(\gamma_{i} \mathcal{T}_{+}+\mathcal{T}_{+}{ }^{2}\right) q_{i}^{2}-\left(\frac{\Delta}{\mathcal{T}_{-}^{2}}+\theta^{2}\right)\left(U^{\prime}\left(q_{i+1}-q_{i}\right)^{2}+p_{i}^{2}\right)
\end{aligned}
$$

where $\Delta=\max _{i}\left|T_{i}-T_{i+1}\right|, \mathcal{T}_{+}=\max _{i} T_{i}, \mathcal{T}_{-}=\max _{i} T_{i}$ and $\theta=\max _{i}\left|\theta_{i}\right|$.

From the assumptions (37) on the potentials and (107), we see that as soon

$$
\inf _{i}\left\{\frac{\delta}{\gamma_{i} \mathcal{T}_{+}+\mathcal{T}_{+}^{2}}, \frac{\gamma_{i}}{8 \mathcal{T}_{+}}\right\}>b \quad \text { and } \quad \inf _{i}\left\{b \delta, \frac{\gamma_{i}}{8 \mathcal{T}_{+}}\right\}>\frac{\Delta}{\mathcal{T}_{-}^{2}}+\theta^{2}
$$

then

$$
\Phi(\underline{p}, \underline{q}) \geq \sum_{i=1}^{N} c_{1}\left[p_{i}^{2}+V\left(q_{i}\right)+U\left(q_{i}-q_{i+1}\right)\right]-c_{2},
$$


for some constants $c_{1}>0, c_{2}$. This is enough to conclude that $\Phi$ diverges at infinity and that the large deviation principle follows from (103) (see [39]).

From (109), one can also derive an upper bound on the total current (7)

$$
\Phi(\underline{p}, \underline{q})+c_{2} \geq \alpha N|J(\underline{q}, \underline{p})|
$$

for some constant $\alpha>0$. From (103), we deduce the exponential bound (38) on the current.

Acknowledgments: We thank Christian Maes for useful discussions. The authors acknowledge the support of the ANR LHMSHE.

\section{References}

[1] K. Aoki, J. Lukkarinen and H. Spohn: Energy Transport in Weakly Anharmonic Chains. J. Stat. Phys. 124, 1105-1129 (2006).

[2] L. Bertini, A. De Sole, D. Gabrielli, G. Jona-Lasinio, and C. Landim : Macroscopic Fluctuation Theory for Stationary Non-Equilibrium States. J. Stat. Phys. 107, 635-675 (2002).

[3] L. Bertini, A. De Sole, D. Gabrielli, G. Jona-Lasinio, and C. Landim: Non equilibrium current fluctuations in stochastic lattice gases J. Stat. Phys. 123, no. 2, 237-276 (2006).

[4] T. Bodineau and B. Derrida: Current Fluctuations in Nonequilibrium Diffusive Systems: An Additivity Principle. Phys. Rev.Lett. 92, 180601 (2004).

[5] T. Bodineau and B. Derrida: Cumulants and large deviations of the current through non-equilibrium steady states, C.R. Physique 8, 540-555 (2007).

[6] F. Bonetto, J. Lebowitz and J. Lukkarinen: Fourier's Law for a Harmonic Crystal with Self-Consistent Stochastic Reservoirs, J. Stat. Phys. 116, 783-813, (2004).

[7] J. Bricmont and A. Kupiainen: Towards a derivation of Fourier's law for coupled anharmonic oscillators, Comm. Math. Phys. 274 no. 3, 555-626 (2007).

[8] S. Bruers, C. Maes and K. Netočný: On the validity of entropy production principles for linear electrical circuits. J. Stat. Phys. 129, no. 4, 725-740 (2007).

[9] P. Carmona: Existence and uniqueness of an invariant measure for a chain of oscillators in contact with two heat baths. Stoch. Proc. Appl. 117, 1076-1092 (2007).

[10] J. Deuschel, D. Stroock: Large deviations, Pure and Applied Mathematics, 137 Academic Press (1989).

[11] M.D. Donsker and S.R. Varadhan: Asymptotic evaluation of certain Markov process expectations for large time, I Comm. Pure Appl. Math, 28:1-47 (1975). 
[12] M.D. Donsker and S.R. Varadhan: Asymptotic evaluation of certain Markov process expectations for large time, III Comm. Pure Appl. Math. 29, no. 4, 389-461 (1976).

[13] J.-P. Eckmann, C.-A. Pillet, L. Rey-Bellet: Non-equilibrium statistical mechanics of anharmonic chains coupled to two heat baths at different temperatures. Commun. Math. Phys. 201, 657-697 (1999).

[14] J-P. Eckmann, C-A. Pillet, L. Rey-Bellet:Entropy production in Non-linear, Thermally Driven Hamiltonian Systems. J. Stat. Phys. 95, 305-331 (1999).

[15] J.-P. Eckmann, M. Hairer: Non-Equilibrium Statistical Mechanics of Strongly Anharmonic Chains of Oscillators. Commun. Math. Phys. 212, 105-164 (2000).

[16] D. J. Evans, E. G. D. Cohen, and G. P. Morriss: Probability of second law violations in shearing steady states. Phys. Rev. Lett. 71, 2401-2404 (1993).

[17] G. Eyink, J. Lebowitz and H. Spohn: Microscopic Origin of Hydrodynamic Behavior: Entropy Production and the Steady State "CHAOS/XAOC" SovietAmerican Perspectives on Nonlinear Science, David K. Campbell, editor. (1990).

[18] G. Gallavotti and E. G. D. Cohen: Dynamical Ensembles in Nonequilibrium Statistical Mechanics. Phys. Rev. Lett. 74, 2694-2697 (1995).

[19] G. Gallavotti and E. G. D. Cohen: Dynamical ensembles in stationary states. J. Stat. Phys. 80, 931-970 (1995).

[20] R. Kubo, K. Matsuo, and K. Kitahara: Fluctuations and relaxation of macrovariables. J. Stat. Phys. 9, 51-95 (1973).

[21] J. Kurchan: Fluctuation Theorem for stochastic dynamics. Journal of Physics A 31, 3719 (1998).

[22] J. Kurchan: Gallavotti-Cohen Theorem, Chaotic Hypothesis and the Zero-Noise Limit, J. Stat. Phys. 128, No 6, 1307-1320 (2007).

[23] J.L. Lebowitz and H. Spohn: A Gallavotti-Cohen-type symmetry in the large deviation functional for stochastic dynamics. J.Stat.Phys. 95, 333-365 (1999).

[24] R. Lefevere: On the local space-time structure of non-equilibrium steady states. J. Stat. Mech. P01004 (2007).

[25] R. Lefevere and A. Schenkel: Normal Heat Conductivity in a strongly pinned chain of anharmonic oscillators. J. Stat. Mech. L02001 (2006).

[26] C. Maes: Fluctuation theorem as a Gibbs property. J.Stat.Phys. 95, 367-392 (1999).

[27] C.Maes, K. Netočný and M.Verschuere: Heat conduction networks. J.Stat.Phys. 111,1219-1244 (2003)

[28] C. Maes and K. Netočný: Minimum entropy production principle from a dynamical fluctuation law. J. Math. Physics 48, 053306 (2007). 
[29] C. Maes and K. Netočný: The canonical structure of dynamical fluctuations in mesoscopic nonequilibrium steady states. arXiv:0705.2344

[30] C. Maes, K. Netočný and B. Wynants: Steady state statistics of driven diffusions. arXiv:0708.0489

[31] C. Maes, K. Netočný and B. Wynants: On and beyond entropy production; the case of Markov jump processes., arXiv:0709.4327

[32] I. Prigogine: Introduction to Non-equilibrium Thermodynamics. WileyIntersciences, New-York (1962).

[33] L. Rey-Bellet, Ergodic properties of Markov processes, Lecture Notes of the 2003 Grenoble Summer School on Open Quantum Systems (2003).

[34] L. Rey-Bellet and L.E. Thomas: Exponential Convergence to Non-Equilibrium Stationary States in Classical Statistical Mechanics. Comm. Math. Phys. 225, 305-329 (2002).

[35] L. Rey-Bellet and L.E. Thomas: Fluctuations of the entropy production in anharmonic chains, Ann. Henri Poincaré 3, 483-502 (2002).

[36] H. Spohn: Large scale dynamics of interacting particles, Springer-Verlag (1991).

[37] H. Spohn: The phonon Boltzmann equation, properties and link to weakly anharmonic lattice dynamics. J. Stat. Phys. 124, 10411104 (2006).

[38] D. W. Stroock and S.R.S. Varadhan, On the support of diffusion processes with applications to the strong maximum principle., Proceedings of the Sixth Berkeley Symposium on Mathematical Statistics and Probability (Univ. California, Berkeley, Calif., 1970/1971), Probability Theory (Berkeley, Calif.) vol. III, Univ. California Press (1972),

[39] L. Wu: Large and moderate deviations and exponential convergence for stochastic damping Hamilton systems, Stoch. Proc. Appl., 91 (2), 205-238 (2001). 